\title{
FRACTAL ANALYSIS OF TRABECULAR BONE PATTERN IN THE MANDIBLE AS AN INDICATOR OF OSTEOPOROSIS IN WOMEN - A CLINICAL STUDY
}

\author{
Vijayalakshmi K, Krithika C L, Raghuram P H, Kannan A \\ Department of Oral Medicine and Radiology, SRM Dental College, Bharathi Salai, Ramapuram, Chennai, Tamilnadu - 600 089. \\ Email: vlakshmi754@gmail.com \\ Received: 22 September 2017, Revised and Accepted: 04 November 2017
}

\section{ABSTRACT}

Objectives: The objectives of this study were to estimate and compare the measurement of trabecular bone pattern in the mandible of normal and osteoporotic volunteers.

\begin{abstract}
Methods: A 43 female volunteers were selected as osteoporotic $(n=43)$ group and 30 as normal $(n=30)$ group with age ranging from 25 to 60 years were enrolled based on bone mineral densitometer (BMD) in the calcaneus bone. A detailed case history followed by digital periapical radiograph was performed. The mandibular trabecular bone pattern in these volunteers was determined using Image J software, after standardizing the pixel size and locations of the region of interest for three different regions between the two groups. Statistical analysis using independent $\mathrm{t}$-test and Pearson coefficient was performed.
\end{abstract}

Results: Results showed a significant difference in mean BMD values between the groups ( 0.52 in normal and -3.22 in osteoporotic). There are no significant differences in mean fractal dimension values between the groups ( 0.83 in normal and 0.82 in osteoporotic). Pearson correlation coefficient shows no significant correlation between the groups at three sites $(\mathrm{p}>0.001)$.

Conclusion: Although trabecular bone microstructure on an intraoral radiograph plays a key role in defining osteoporosis, the present study did not show any significant difference in its architecture between normal and osteoporotic individuals as defined by BMD. Therefore, further studies should be performed using better-standardized resolution strategies and different estimation methods to gain more insight.

Keywords: Dental radiograph, Osteoporosis, Densitometry, Trabecular bone, Fractals.

(c) 2018 The Authors. Published by Innovare Academic Sciences Pvt Ltd. This is an open access article under the CC BY license (http://creativecommons. org/licenses/by/4. 0/) DOI: http://dx.doi.org/10.22159/ajpcr.2018.v11i2.22719

\section{INTRODUCTION}

Osteoporosis is a skeletal disorder characterized by compromised bone strength predisposing a person to an increased risk of fracture [1]. Measurements of bone mineral densitometer (BMD) are done by various methods such as dual-energy X-ray absorptiometry (DEXA) scan, bone biopsy, and other adjuncts include quantitative ultrasound calcaneal (QUS), computed tomography (CT), and magnetic resonance imaging. Numerous studies have shown that DEXA scan though being the gold standard method for detecting osteoporosis has its own limitations which prevent it from being used in the mass screening of osteoporosis. Compared to DEXA, QUS offers wider accessibility, portability, ease of handling, economical, and does not emit ionizing radiation [2].

Trabecular bone has a branching pattern in which bone remodeling is more and is the significant indicator of osteoporosis [3]. However, osteoporosis is not only characterized by a decrease in density but also by structural changes in the architecture of trabecular bone. The inner aspect of alveolar bone appears spongy having spicules, trabecular, and lamella which give its typical structure. Lattice pattern has an accurate expression of the internal bony medullary cavity on intraoral periapical radiograph [4]. Many studies have been carried out to determine the quality of alveolar bone of which fractal analysis is accurate, easily available, and economical [5]. In fractal dimension (FD), morphometric analysis along with mathematical processing is done using a radiographic image. There are many counting algorithms in the fractal analysis, of this box-counting method was used to analyze the trabecular pattern.

Digital radiography made with digital sensors facilitates image manipulation, reduced patient exposure, relatively inexpensive, and viewing the minute details of the image. These images are stored on the computer and shared for the benefit of patient management [6].
Although many studies have been performed using fractal analysis to determine osteoporosis, many authors have not demonstrated the roles of standardized resolution and location of the region of interest (ROI) [7]. With this background, the aim of our study was to compare and estimate the measurements of the trabecular bone using standardized pixel dimension and defined regions of interest in both the groups. The present study is unique wherein osteoporosis screening was done using calcaneus BMD instead of DEXA to enroll the study participants.

\section{METHODS}

\section{Study group}

The study was performed in the oral medicine and radiology department which was approved by Ethical Committee Review Board and informed written patient consent was obtained from all the subjects. A mass screening for osteoporosis was done for 1 week using QUS device in women with age ranging between 25 and 60 years. A total of 43 were in the osteoporotic group in which 13 were excluded: 10 due to systemic diseases and medications and 3 were not willing to participate in the study and 30 were included in the control group. Patients with systemic conditions such as a chronic renal disease, anemia, hyperthyroidism, long-term steroid medications, Paget's disease, and dental conditions such as periapical and periodontal pathologies were excluded.

\section{BMD evaluation}

BMD evaluation was done using the bone densitometer CM-200 machines at calcaneus region. It is based on the measurement of ultrasound pulse penetration. For estimation, the participants were recommended to place the calcaneus bone of the right leg in the foot compartment of CM-200 devices (Fig. 1). The software of the CM-200 devices will evaluate the BMD of the calcaneus bone. The measurement results were determined by T-score criteria set by the World Health Organization (WHO) which was graphically presented on the device 
monitor. T-score $>-1$ is normal; T-score between -1.1 and -2.5 is osteopenia; and T-score $\leq-2.5$ is osteoporosis [8]. The volunteers were then subjected to oral examination to evaluate the dental status.

\section{Radiographic evaluation}

The Satelec Acteon X-ray machine with exposure parameters of $0.250 \mathrm{~mA}$ and $70 \mathrm{kVp}$ was used. PSP sensor was used to record the digital periapical radiograph using paralleling technique. The films were processed and the images were stored in BMP (Bitmap) format. The images were digitized by 8-bit image type and $300 \mathrm{dpi}$ spatial resolution by a logarithmic algorithm. All images were analyzed using Image J version $1.32 \mathrm{j}$, public domain software developed by National Institute of Health (US). A pixel size of $34 \times 30$ was fixed and was used in all the ROI. Three regions of interest were selected. The regions of interestinclude: (i) D1-apical area of mandibular premolar tooth, (ii) D2interproximal area between molar and premolar, and (iii) D3-posterior to the last mandibular molar and superior to the inferior alveolar nerve canal. The three ROI selection on the periapical radiograph was done using the criteria of measuring $2.5 \mathrm{~mm}$ from the root apices or mesially or distally [9]. The ROI was cropped to binary image and skeletonized image using white and Rudolph method [10]. The skeletonized image indicates the bone pattern (Fig. 2). This image was then converted into the widths of the square boxes with $2,3,4,6,8,12,16,32$, and 64 pixels. Finally, FD value (D-value) (Fig. 3) were calculated.

\section{Statistical analysis}

Data analysis was performed using SPSS 22.0 software. Independent t-test was used to calculate the mean values between the groups of variables. Pearson correlation coefficient analysis was used to calculate the linear relationship between variables of the two groups.

\section{RESULTS}

Table 1 summarizes the mean BMD and FD of the study groups. The mean BMD T-score in control groups was 0.52 and in study groups was -3.22. The mean FD value in control groups was 0.83 and in study groups was 0.82. A significant difference in the BMD values was found between the groups $(\mathrm{p}<0.001)$.

Table 2 summarizes the FD values of the study subjects according to three sites with no significant differences between the groups ( $>0.001$ ). Table 3 summarizes the correlation coefficient at the three sites between the groups with no significant differences.

\section{DISCUSSION}

The present study aimed at estimating and comparing the measurements of trabecular bone pattern in the mandible of normal and osteoporotic conditions. Osteoporosis is "a systemic skeletal disease characterized by low bone mass and micro-architectural deterioration of bone tissue, with a consequent increase in bone fragility and susceptibility to fractures" [11]. The evaluation of BMD by DEXA scan is the gold standard method for assessing the osteoporotic risk (WHO, 2003). Most of the studies have used calcaneal BMD less frequently in comparison to DEXA [7]. Studies on calcaneus BMD have a great advantage compared to other skeletal bones, and the measurements are not affected by local factors such as spinal deformation and fractures [12]. A study by Martini et al. proved that calcaneus BMD is an accurate and cost-effective technique in the diagnosis of osteoporosis [13]. Therefore, the present study, to the best of our knowledge, is the first one in which osteoporosis screening was done using calcaneus BMD instead of DEXA to determine the study group. None of the study participants were known osteoporotic.

Lindh et al. showed that the bone remodeling is more extensive in the trabecular bone than in the compact bone which is the manifestation of osteoporosis. It is not clear what structure give rise to the trabecular pattern. A study by Bender et al. showed that the posterior regions of mandible did not alter in radiographic appearance of trabeculae when influenced by the muscle activity or the occlusal forces [14]. Most of the clinical studies have not taken into consideration the odds of trabecular

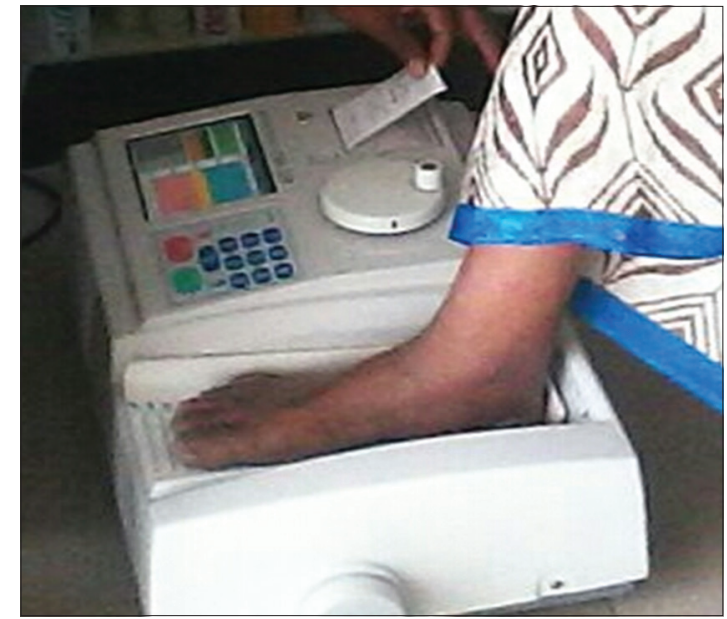

Fig. 1: Bone mineral densitometer CM-200 device
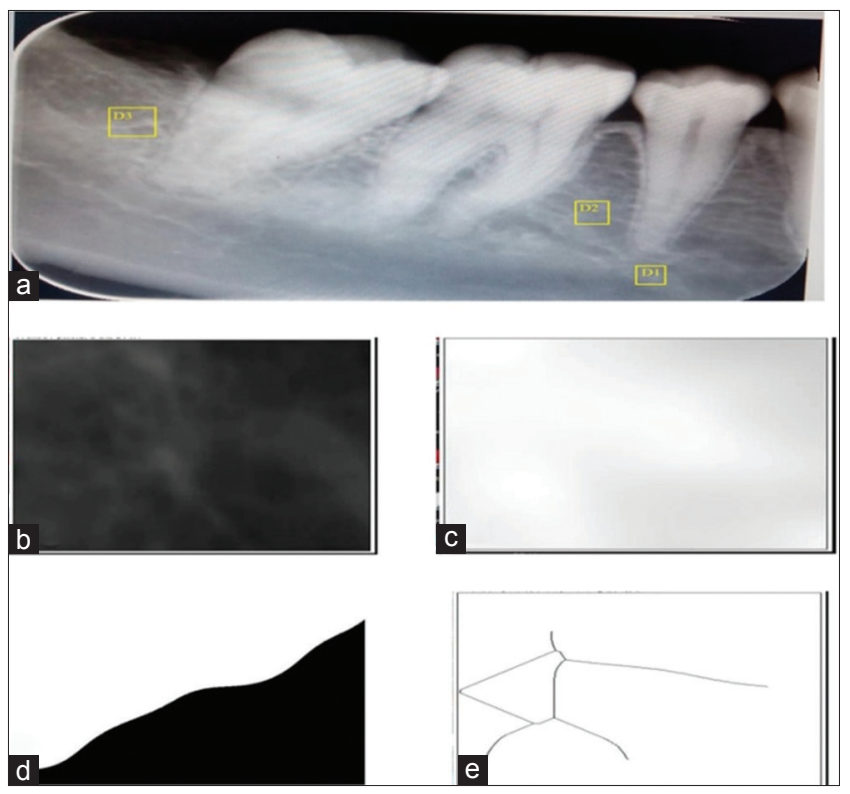

Fig. 2: (a) Region of interest, (b) blurred image, (c) grayscale image, (d) binary image, and (e) skeletonized image

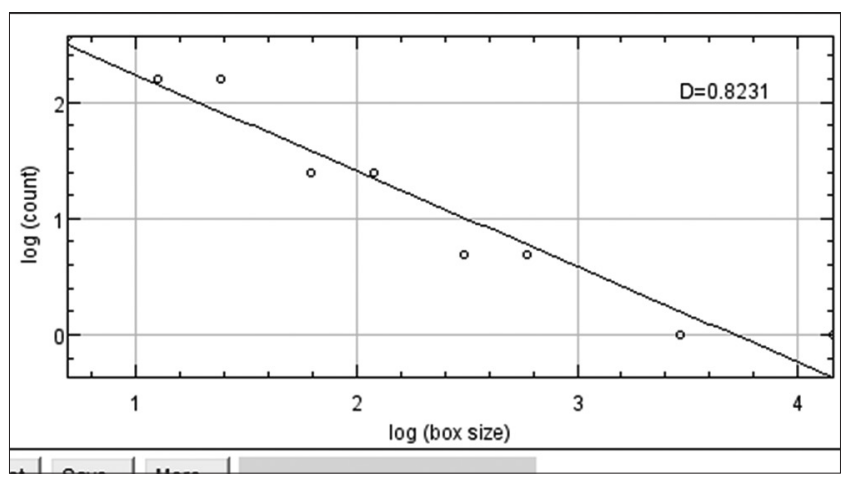

Fig. 3: Shows fractal dimension values calculated from the slope of the line fitted to the data points

bone architecture changes by anatomical variations, various imaging methods, and different estimation methods for measuring FD. Our study is the first in the literature which has aimed at standardizing the pixel size at $34 \times 30$ and location of ROI by taking three different sites: D1, D2, and D3, respectively. 
Table 1: Mean BMD and FD values of the study groups

\begin{tabular}{|c|c|c|c|c|}
\hline Variables & Group & Mean \pm SD & $\mathbf{T}$ & $\mathbf{p}$ \\
\hline \multirow[t]{2}{*}{ FD: D1 } & Osteoporotic & $0.82967 \pm 0.099116$ & 0.254 & 0.800 \\
\hline & Normal & $0.83633 \pm 0.104138$ & & \\
\hline \multirow[t]{2}{*}{ FD: D2 } & Osteoporotic & $0.84367 \pm 0.131477$ & 0.123 & 0.903 \\
\hline & Normal & $0.84733 \pm 0.097872$ & & \\
\hline \multirow[t]{2}{*}{ FD: D3 } & Osteoporotic & $0.82367 \pm 0.128129$ & 0.337 & 0.738 \\
\hline & Normal & $0.83433 \pm 0.116993$ & & \\
\hline \multirow[t]{2}{*}{ BMD } & Osteoporotic & $-3.22333 \pm 0.550350$ & 12.377 & $<0.001$ \\
\hline & Normal & $0.52500 \pm 1.564738$ & & \\
\hline \multicolumn{5}{|c|}{$\begin{array}{l}\mathrm{N}: 30 \text { Normal group, } \mathrm{N}=30 \text { osteoporotic group, FD: Fractal dimension, } \\
\mathrm{BMD} \text { : Bone mineral densitometry. D1: Apical region of mandibular premolar } \\
\text { tooth, D2: Interproximal area between molar and premolar, D3: Posterior } \\
\text { to the last mandibular molar and superior to the inferior alveolar nerve } \\
\text { canal, SD: Standard deviation }\end{array}$} \\
\hline \multicolumn{5}{|c|}{ Table 2: FD values of the study subjects according to three sites } \\
\hline Site & Normal - FD values & \multicolumn{2}{|c|}{ Osteoporosis - FD values } & $\mathbf{p}$ \\
\hline D1 & 0.83 & \multicolumn{2}{|l|}{0.82} & 0.800 \\
\hline $\mathrm{D} 2$ & 0.84 & \multicolumn{2}{|l|}{0.84} & 0.903 \\
\hline D3 & 0.83 & 0.82 & & 0.738 \\
\hline
\end{tabular}

FD: Fractal dimension, D1: Apical region of mandibular premolar tooth, D2: Interproximal area between molar and premolar, D3: Posterior to the last mandibular molar and superior to the inferior alveolar nerve canal

Table 3: Correlation coefficient between the three sites

\begin{tabular}{llll}
\hline Fractal dimension & All & Osteoporotic & Normal \\
\hline FD: D1 & & & \\
$\quad$ Correlation & 0.093 & -0.330 & 0.291 \\
p & 0.479 & 0.075 & 0.118 \\
FD: D2 & & & \\
$\quad$ Correlation & 0.011 & -0.232 & 0.101 \\
$\quad$ Significant (2-tailed) & 0.932 & 0.217 & 0.595 \\
FD: D3 & & & \\
$\quad$ Correlation & 0.168 & 0.203 & 0.312 \\
$\quad$ Significant (2-tailed) & 0.200 & 0.282 & 0.093 \\
\hline
\end{tabular}

FD: Fractal dimension, D1: Apical region of mandibular premolar tooth, D2: Interproximal area between molar and premolar, D3: Posterior to the last mandibular molar and superior to the inferior alveolar nerve canal

The use of digital periapical radiograph helps in the assessment of trabecular bone which could be a cost-effective tool for identifying individuals at risk of osteoporosis. Several methods have been developed to estimate the trabecular bone density from radiographs; fractal analysis holds the greatest value in determining the trabecular complexity and bone structure. Amer et al. stated that different regions in periapical radiograph revealed significant differences in the FD values [15]. Yasar et al. suggested that FD value did not show significant differences in the trabecular bone pattern [16]. These results are conflicting. Hence, the present study was performed using standardized resolution and ROI. Several attempts have been made for the reliability of FD estimation with different algorithmic methods. Box-counting method, despite being the commonly used one has several limitations like magnification factor [9]. Matheus et al. showed that FD value did not change by tile counting method. Images influenced by various spatial resolutions produce different FD values [3]. Cha et al. and Geraets and van der Stelt mentioned that the use of different methods of estimation for fractal analysis could be a problem, as its diagnostic accuracy becomes questionable $[17,18]$. The results can change based on the techniques used to determine FD. Brewer demonstrated the roles of resolution and quantization as limiting factors in the estimation of FD [19]. Many studies have shown that the problem in the fractal analysis of binary images is the effect of noise [9]. Since no estimation methods work well universally, the relative discrepancies of all estimating techniques need to gain insights [20]. In our study using digital images, FD values did not change when spatial resolution is the same by box-counting method. Therefore, each imaging modality has its own non-linear artifacts such as resolution and quantization. FD analysis of cone beam CT images warrants further investigations, as only one study has used this imaging modality [7].

Our study has merits and drawbacks. Due to the strength, we have standardized the pixel size and locations of ROI at three different sites in calculating FD values. This suggests that each site is not influenced by local factors such as occluding teeth and masticator forces. Consequently, the limitations such as roles of resolution, quantization, and different methods of estimation of FD values were questionable. The imaging modality is also a factor since we used two-dimensional (2D) images which have noise and sampling frequency as drawbacks. Therefore, three-dimensional (3D) images should be used for better quality of images and determination of FD values.

\section{CONCLUSION}

FD values of the trabecular bone assessed by digital images, boxcounting method, standardized pixel size, and three different ROI did not show any significant differences between osteoporotic and normal volunteers. Further, multi-centric studies should be performed with larger sample size, 3D techniques along with the measurements of biochemical markers such as serum alkaline phosphatase and calcium to give an overview of the bone turnover, which could also be costeffective and simple. This can give a better insight into using QUS and periapical radiographs as osteoporosis indicators in mass screening.

\section{ACKNOWLEDGMENTS}

This study was supported by the faculty of Oral Medicine and Radiology Department, SRM Dental College, Ramapuram, Chennai, Tamil Nadu, India. The contribution of study participants is gratefully acknowledged.

\section{AUTHORS CONTRIBUTION:}

1. Dr. Vijaya Lakshmi K, Post Graduate, SRM Dental College

2. Dr. Krithika. C.L, MDS, Reader, SRM Dental College

3. Dr. P.H. Raghuram, MDS, Head of the Department, SRM Dental College 4. Dr. Kannan. A, MDS, Associate Professor, SRM Dental College

\section{REFERENCES}

1. Kumar AV, Joseph AK, Gokul G, Alex MP, Naveena G. Clinical outcome of calcium, Vitamin D3, and physiotherapy in osteoporotic population in the Nigari's district. Int J Pharm Pharm Sci 2016;8:157-60.

2. Laugier P. An overview of bone sonometry. Int Congr Ser 2004; 1274:23-32.

3. Oliveira ML, Pedrosa EF, Cruz AD, Haiter-Neto F, Paula FJ, Watanabe PC. Relationship between bone mineral density and trabecular bone pattern in postmenopausal osteoporotic Brazilian women. Clin Oral Invest 2012;17:1847-53.

4. Jett S, Shrout MK, Mailhot JM, Potter BJ, Borke JL. An evaluation of the origin of trabecular bone patterns using visual and digital image analysis. Oral Surg Oral Med Oral Pathol Oral Radiol Endod 2004;98:598-604.

5. Ergün S, Saraçoglu A, Güneri P, Ozpinar B. Application of fractal analysis in hyperparathyroidism. Dentomaxillofac Radiol 2009;38:281-8.

6. Chen SK, Oviir T, Lin CH, Leu LJ, Cho BH, Hollender L. Digital imaging analysis with mathematical morphology and fractal dimension for evaluation of periapical lesions following endodontic treatment. Oral Surg Oral Med Oral Pathol Oral Radiol Endod 2005;100:467-72.

7. Leite AF, de Souza Figueiredo PT, Caracas H, Sindeaux R, Guimarães AT, Lazarte L, et al. Systematic review with hierarchical clustering analysis for the fractal dimension in assessment of skeletal bone mineral density using dental radiograph. Oral Radiol 2014;31:1-13.

8. Kullenberg R, Falch JA. Prevalence of osteoporosis using bone mineral measurements at the calcaneus by dual X-ray and laser (DXL). Osteoporos Int 2003;14:823-7.

9. Carranza FA, Newman MG. Clinical Periodontology. $8^{\text {th }}$ ed. Philadelphia, PA: WB Saunders; 1996. p. 297-313.

10. White SC, Rudolph DJ. Alterations of the trabecular pattern of the jaws 
in patients with osteoporosis. Oral Surg Oral Med Oral Pathol Oral Radiol Endod 1999;88:628-35.

11. Miraçi M, Demeti A, Ylli Z, Kelliçi S, Tarifa D. The cost-effectiveness of ibandronate and alendronate for the treatment of osteoporosis in a specialized clinic in Tirana. Int J Pharm Pharm Sci 2015;7:207-11.

12. Vogel JM, Wasnich RD, Ross PD. The clinical relevance of calcaneus bone mineral measurements: A review. Bone Miner 1988;5:35-58.

13. Martini G, Valenti R, Gennari L, Salvadori S, Galli B, Nuti R. Dual $\mathrm{X}$-ray and laser absorptiometry of the calcaneus: Comparison with quantitative ultrasound and dual-energy X-ray absorptiometry. J Clin Densitom 2004;7:349-54.

14. Bender IB, Seltzer S. Roentgenographic and direct observation of experimental lesions in bone: II 1961. J Endod 2003;29:707-12.

15. Amer ME, Heo MS, Brooks SL, Benavides E. Anatomical variations of trabecular bone structure in intraoral radiographs using fractal and particles count analyses. Imaging Sci Dent 2012;42:5-12.
16. Yasar F, Akgünlü F. The differences in panoramic mandibular indices and fractal dimension between patients with and without spinal osteoporosis. Dentomaxillofac Radiol 2006;35:1-9.

17. Cha SY, Han WJ, Kim EK. Usefulness of fractal analysis for the diagnosis of periodontitis. Korean J Oral Maxillofac Radiol 2001;31:35-42.

18. Geraets WG, van der Stelt PF. Fractal properties of bone. Dentomaxillofac Radiol 2000;29:144-53.

19. Brewer J, Girolamo LD. Limitations of fractal dimension estimation algorithms with implications for cloud studies. Atmos Res 2006;82:433-54.

20. Chappard C, Brunet-Imbault B, Lemineur G, Giraudeau B, Basillais A, Harba R, et al. Anisotropy changes in post-menopausal osteoporosis: Characterization by a new index applied to trabecular bone radiographic images. Osteoporos Int 2005;16:1193-202. 